Topical Review

\title{
On the Strategy of Kinetic Discrimination of Amino Acid Transport Systems
}

\author{
Halvor N. Christensen \\ Department of Biological Chemistry, The University of Michigan Medical School, Ann Arbor, Michigan 48109
}

\section{Introduction}

It was for renal epithelial transport that the logical conclusion was first reached that different agencies serve for the transport of cationic and zwitterionic amino acids [4]. Such partitions according to molecular charge have usually proved valid for other cells and tissues of the higher animal, given that attention is applied to identify the charge retained by the amino acid during transport, and also that allowance is made for the possibility that the transport system may have its charge changed by protonation or deprotonation (see review on the influence of molecular charge on the route of amino acid transport, ref. [7]). In the early sixties it came to be appreciated that a single agency would not, however, account for the transport of the zwitterionic amino acids, and thereafter the significant discrimination was made between the $\mathrm{Na}^{+}$-dependent and $\mathrm{Na}^{+}$-independent agencies [17]. Each of these two transport systems showed other important distinguishing characteristics, for example, the extent to which inward transport was quickly offset by outward transport [17]. Furthermore, other limited tests suggested homogeneity of each of these two components in the Ehrlich cell. Already by the time we reported that result, it could be sensed that the $\mathrm{Na}^{+}$-dependent component probably represented more than one system-note the change in title in going from our preliminary [16] to our final [17] report. An important feature of the latter report was the finding that parallel transport by two or more systems tended to be characteristic for any amino acid because of the heavy overlap in their amino acid selectivity. This discovery led ultimately to

Key Words model substrates $\cdot$ molecular charge and transport - $\mathrm{Na}^{+}$dependence $\cdot$ negative cooperativity $\cdot$ nonsaturable migration $\cdot$ transport regulation conceptions as to how systems might cooperate in maintaining steady-state amino acid distributions and flows [8].

The second component of $\mathrm{Na}^{+}$-dependent transport, namely System ASC, was easily recognized as different from System A in erythrocytes, even though a more difficult differentiation succeeded first in the Ehrlich cell [10]. In retrospect [15], the discrimination of ASC should also have been easier for cells cultured in amino acid-rich media, a condition under which System A is relatively repressed. Of the two systems, System ASC is ordinarily the more conspicuous and undoubtedly quite as important in the amino acid environment of the intact organism, where $\mathrm{A}$ is also characteristically repressed. In further retrospect, these two $\mathrm{Na}^{+}$-dependent systems are so different in other biological features that the persistence of the idea of a simple $\mathrm{A} / \mathrm{L}$ classification long after our laboratory corrected it $[10]$ has been mystifying. Subsequently two other $\mathrm{Na}^{+}$-dependent systems rather more like System A were added, one serving for glycine and sarcosine $[9,23]$ and the other for glutamine, histidine and asparagine [14]; also a $\beta$-system tolerating a larger separation between the charged aminium and anionic groups of the dipolar amino acids, including taurine [5]. Even then we do not mean to suggest that the list is finished. For example, the principal $\mathrm{Na}^{+}$-dependent transport system at the epithelial brush borders seems sadly not yet to fit well into any well-characterized categories, although it resembles ASC more than A. Perhaps because of severe overlaps in amino acid selectivity between wide-scope systems, also perhaps even more because of unusual susceptibility of this tissue to unstirred-layer effects, a reluctance may have been encountered to apply in this area the full kinetic strategies of analog inhibition analysis, for which I had offered a summary in 1966 [6]. 


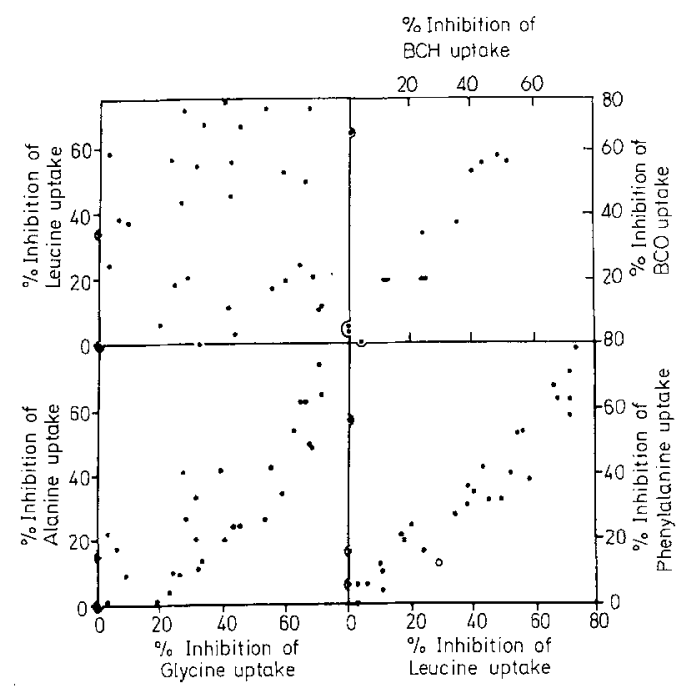

Fig. 1. Correlation between the inhibitory actions of various amino acids on the uptake of pairs of amino acids by an isolated cell in suspension. Upper left, no significant correlation is evident in the inhibition of thirty-odd amino acids on the uptake by the Ehrlich cell of glycine (abscissa) and of leucine (ordinate). Lower left, correlation is evident for some of these amino acids between the inhibition of the uptake of glycine and alanine by the same cell. Lower right, rather more extensive correlation is seen in their inhibition of the uptake by the same cell of leucine and phenylalanine (these 3 diagrams represent data from ref. (17)). Upper right, correlation between the inhibition by 13 amino acids of the uptake by the human erythrocyte of the bicycloheptane $(\mathrm{BCH})$ and the bicyclooctane amino acid [22]. This plot indicates that these two bicyclic amino acids fail to discriminate between the two known components of their $\mathrm{Na}^{+}$-independent uptake by this cell. A similar comparison using leucine and tryptophan as transport substrates uncovers the heterogeneity [22]

\section{Heterogeneity also in the $\mathrm{Na}^{+}$-Independent Component}

It is interesting that erythrocytes provided the first opportunities to recognize the heterogeneity also of the $\mathrm{Na}^{+}$-independent component of dipolar amino acid transport, when System T, a variant of System $\mathrm{L}$ preferring tryptophan, tyrosine and phenylalanine, was uncovered in the human red cell [19]. Had we fully applied our own advice for detailed inhibition analysis [6], we should no doubt have discovered in our 1967 study [11] the presence in the pigeon erythrocyte of a transport system utterly different from System L, which is the system whose special properties have so far dominated $\mathrm{Na}^{+}$-independent transport of dipolar amino acids. The new system prefers threonine, cysteine, serine and alanine, as a preliminary list, and approaches its steady state much more slowly [22]. Species variants of this new system had unknowingly already been discovered in the erythrocytes of some but not all sheep as a cysteine-transporting system [27] and more recently in the erythrocytes of some but not all horses [12]. In amino acid selectivity, the discoverers were logically struck by the resemblance of this system to $\mathrm{Na}^{+}$-dependent System ASC, and therefore proposed that it arose through the loss of $\mathrm{Na}^{+}$-dependency by that system during red blood cell maturation in most members of these species. The congenital absence of this system in some sheep and in some horses facilitated its discovery.

We were puzzled as to how System ASC could retain its usual amino acid selectivity in the absence of $\mathrm{Na}^{+}$, considering the crucial role played by $\mathrm{Na}^{+}$ in generating the amino acid receptor site. We were led accordingly to see [22] that the contaminant of System L in the pigeon red cell we were then studying, encountered while examining for System $\mathrm{T}$ and for another $\mathrm{Na}^{+}$-independent system found in rat hepatocytes [24], was undoubtedly a species variant of the system thus seen in the erythrocytes of some sheep [27] and some horses [12]. Because the red blood cell of the pigeon is nucleated, it undoubtedly resembles functionally some of the precursors of the unnucleated mammalian red blood cell; indeed our earlier analyses of rabbit reticulocytes appear to be compatible with the presence there also of the new $\mathrm{Na}^{+}$-independent system [22]. These findings indicate that this $\mathrm{Na}^{+}$-independent system is a fundamental biological entity of probably wide occurrence, rather than an exclusive product of the terminal differentiation of mammalian erythrocytes.

The selection of a designation for this system proceeded while a number of us were developing a joint communication [2] to regularize among ourselves such designations for the amino acid transport systems as are tabulated in the adjacent glossary. Ultimately this plan came to include a proposal by Gazzola and Guidotti that lowercase letters might be used to designate $\mathrm{Na}^{+}$-independent transport systems, where the same capital letter abbreviation has already been selected for a similar $\mathrm{Na}^{+}$-dependent system. The glossary illustrates this naming plan. It shows the use of $\mathrm{X}^{-}$and $\mathrm{x}^{-}$for $\mathrm{Na}^{+}-$ dependent and $\mathrm{Na}^{+}$-independent transport systems for the anionic amino acids. As an extension of this plan, Young and his associates and we in this laboratory have agreed to designate the new $\mathrm{Na}^{+}$-independent system as System asc, provisional to completion of the evidence that we are indeed dealing as we suppose with variants of a generic $\mathrm{Na}^{+}$-independent system, characterized at first by the similarity of its amino acid selectivity to that of $\mathrm{Na}^{+}$-dependent System ASC. Limits to that similarity have in the meantime surfaced [22]. I suggest that to signal the discrimination in reading aloud the designations asc and ASC, the two be differentiated by use of the adjectives, $\mathrm{Na}^{+}$-independent and $\mathrm{Na}^{+}$-dependent. 


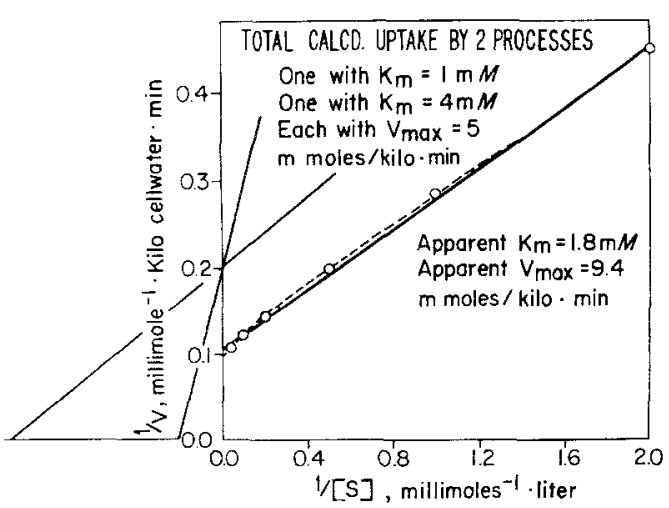

Fig. 2. Lineweaver-Burk plot calculated for uptake by the sum of two processes with the indicated parameters. Note that the parameters would need to be much more greatly separated for clear proof by this plot for mediation of uptake by more than a single transport system. The diagram also illustrates that extrapolation of the plot of the abscissa yields apparent parameters quite unlike the real ones assumed for the calculation. Reproduced with permission from Christensen (1975). Biological Transport, second edition, W.A. Benjamin, Inc., Reading. Mass., p. 140

The 1966 essay [6] on the discrimination of membrane transport systems did not make a recommendation that now seems important, given that a $\mathrm{Na}^{+}$-independent System asc can have an amino acid selectivity quite similar to that of $\mathrm{Na}^{+}$-dependent System ASC. An effort at discrimination of transport systems based like that illustrated in the correlation plots of Fig. 1 purely on analog-inhibition analysis may well yield confusing results if inhibition analysis were not applied separately to the $\mathrm{Na}^{+}$-dependent and the $\mathrm{Na}^{+}$-independent components. One would for example get the impression that System ASC were only partially $\mathrm{Na}^{+}$-dependent in the pigeon red blood cell, because of the coexistence of System ASC and asc in that cell. Hence I add here the recommendation, already widely observed: First divide uptake between $\mathrm{Na}^{+}-$ dependent and $\mathrm{Na}^{+}$-independent; then proceed to analog inhibition analysis. Let me continue then otherwise to update the 1966 essay by emphasizing the following recommendations:

1. One should, I think, be prepared to test a wide variety of amino acid analogs as potential competitive inhibitors in delineating one or more possibly new routes of transport. Further, one should be prepared as I will discuss below occasionally to reverse their roles as transport substrate and as inhibitor. Too sparing a choice of analogs may fail. Because my colleagues and I were afforded a generous interval before others joined us in our peculiar interest, we had the opportunity to benefit from access to numerous amino acid analogs, natu-
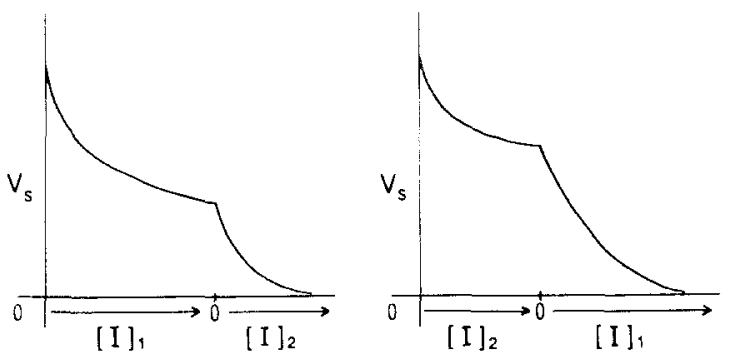

Fig. 3. An idealized representation of the use of two selected analogs for discriminating two independent routes of transport of a test substrate. A prior test of the influx of the test substrate as a function of its concentration may have shown either a precise rectangular hyperbola or ambiguity as to whether more than one rectangular hyperbola was shown. One or more analogs have shown ambiguity on the same question, but progressive changes in molecular structure have led to a pair of analogs each of which eliminates competitively an independent component of uptake

ral and unnatural. Our motivation furthermore seems to have been a particular curiosity as to the effect of substrate structure on transport, rather than an initial appreciation of the range of analogs that would serve optimally.

2. One should allow for a likelihood that the interaction between two analogs for transport will not prove a simple competition for a homogeneous mediating site at the membrane surface. The evidence for homogeneity has often enough proved ultimately inadequate, so that we are well advised to take from the first a position of challenging the homogeneity.

a. A plot of fairly accurate data for $1 / v$ versus $1 /[S]$, or of another of the linear transformations, may provide a straight line, and yet hide a second or third ultimately distinguishable component of transport (Fig. 2). Correspondingly, a plot of the inhibition of the uptake of $S$ by its analog $I$ can also appear linear and yet include competition for more than one mediating system. One attempts to discover the heterogeneity by showing with additional analogs a stronger response at one concentration range than another, or under otherwise modified conditions. This approach maintains the focus on the question, how does the given amino acid pass the membrane? One seeks step-by-step to identify analogs whose inhibition of uptake is limited as nearly as possible to only part of the uptake; that is, to one of the components of uptake. These inhibitors can then be applied sequentially to yield plots like those shown in Fig. 3. It is well to apply next the inhibitors in the reverse order to evaluate the extent to which an inhibitor might itself gener- 


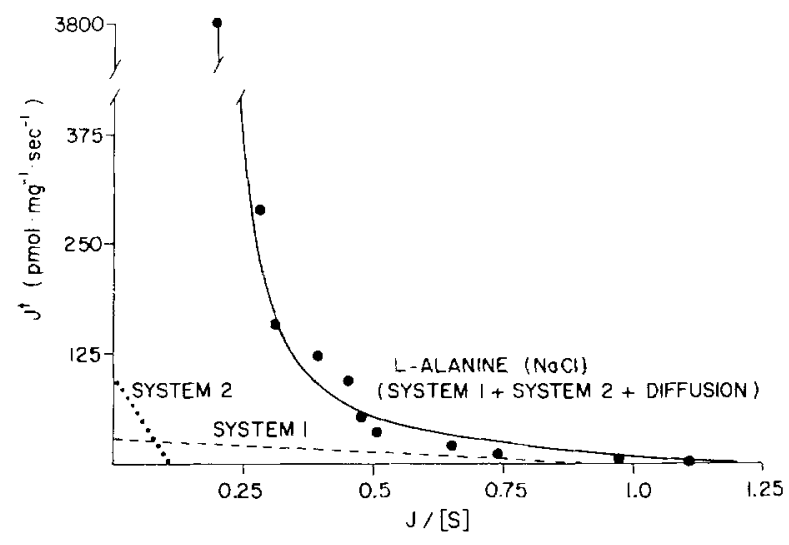

Fig. 4. Hofstee plot of the total alanine influx into rabbit jejunal brush-border vesicles in $\mathrm{Na}^{+}$-containing medium. The computerdirected curve-fitting suggested that the total uptake could be a sum of a linear component, here called diffusion, and saturable uptake by two different systems. The question may be asked (also for a similar plot for phenylalanine uptake) how well the number of mediating systems is proved to be two, or how much more poorly the data would correspond to three distinct mediating systems. Reproduced with permission from B.R. Stevens, H.J. Ross and E.M. Wright (1982) I. Membrane Biol. 66: page 218 (ref. [21] of this paper)

ate an artifactual heterogeneity, for example by modifying the transmembrane potential.

b. One can instead next reduce kinetic complexity and avoid the latter possibility by using the two differential inhibitors selected in Fig. 3 as transport substrates. One shifts attention then to the question, what is the nature of each of the two contributing transport systems? In the course of characterizing each system, one challenges anew the decision that each substrate migrates by a homogeneous route. This approach tends to teach the investigator a chemical basis for selecting amino acid analogs, its ultimate target being the identification for each transport system of what is called a model substrate for that given system. One hopes that the model will serve for occurrences of the system in various cells. Only occasionally will the selected model substrate be an ordinary natural amino acid. Any tendency to avoid artificial analogs can represent a significant blind spot in this approach. Once we know for example that $\mathrm{N}$-methylation of an amino acid may restrict its transport to System A, we can exploit this gain without deploring the possibly low metabolic importance of $\mathrm{N}$-methyl amino acids. The model substrates selected may show the added advantage of resistance to metabolic modification.

3. As a converse to the unreliability of a linear plot as proof of homogeneity of the transport route, one should also question the proposition that a
Lineweaver-Burk curve capable of mathematical resolution into two straight lines necessarily describes two separate mediators. If two, why not three? Figure 4 shows a case where this question can well be asked. Confidence limits were not provided for the half-saturating concentrations of alanine calculated here: $0.32 \mathrm{~mm}$ for the first hypothetical component and $9 \mathrm{~mm}$ for the second. From inspecting the data one wonders if the uncertainty for three mediating systems would be any less. Furthermore, could not a single catalytic agency show a kinetic complexity, e.g. negative cooperativity, causing the line to be bent? The transport of tyrosine and phenylalanine in Bacillus subtilis retains its bent form in isolated mutants (Fig. 5), in the presence of an irreversible inhibitor, or under various other conditions that might be expected to discriminate the supposedly unlike systems [13]. Failures to test for detailed kinetic consistency in the discrimination of transport into independent components merely suggested by bent linear transformations must ultimately lead to serious confusion.

Another over-interpretation of kinetic curves should be considered. Usually a hperbolic form of the plot of $v v s$. $[S]$ for transport will continue upward at a linear slant after completion of the hyperbolic part. The component thus indicated is known as the nonsaturable component. Wilbrandt and Rosenberg pointed out long ago why this component should not be automatically identified with simple diffusion [26]. The migration represented may prove so structurally specific and show so high a temperature coefficient as to raise doubt that it is limited by simple diffusion. The rate may differ among molecules of similar size in a way not logical for diffusion. Selection of a high-affinity analog may bring saturability of the component into an accessible concentration range. For approximate corrections that can be applied to measure the saturable component, however, it may not be important to establish whether the nonsaturable component actually represents mediated transport or some kind of leakiness.

4. One should not assume that the inhibition of the membrane transport of one solute by an analog necessarily identifies transport shared between the two. Instances of an inhibitory action between two amino acids apparently dissociated from shared transport have now multiplied. Noncompetitive inhibition, whether mutual or not, does not suggest identity of transport route. Kinetic proof of shared transport between two analogs is inductive, and hence should involve as many instances as possible. It also requires that the concentrations of each compound saturating its own uptake and that of the ana$\log$ be shown essentially the same, i.e. that the $K_{i}$ for A inhibiting the uptake of B be equal to the $K_{m}$ value for $\mathrm{A}$, and vice versa. Furthermore, because 
these parameters may under real conditions carry large uncertainties, a third test should be added to generate the classical $A B C$ test for shared action of a catalytic site $[1,20]$ : Various dissimilar analogs $C_{1}, C_{2}, C_{3} \ldots$ presumably transported by the same route should each show the same $K_{i}$ value as an inhibitor of the uptake of $A$ and of $B$, respectively. Whether we can prove that transport is not shared in any instance where the inhibition between two analogs is competitive may have only a practical rather than an absolute answer: One of the two may have a $V_{\max }$ for the presumably shared transport so low as to make negligible its flux by the hypothetical common route.

The assumption that inhibition of transport between two amino acids means shared transport may have had excessive acceptance in particular for epithelial transport, partially because of difficulties in securing precision in the kinetic measurements. The $K_{m}$ and $K_{i}$ values obtained tend to be remarkably high, probably because unstirred layer effects are large, especially for the brush-border surface [3]. $K_{i}$ values occasionally have been calculated on the basis of a single test concentration of substrate. For isolated cells and vesicles, and for cultured cell monolayers, unstirred layer effects appear not to distort the sequence of $K_{m}$ and $K_{i}$ values enough to vitiate the results of the $A B C$ test. A historic retarded acceptance of the concept of the participation of multiple transport systems in intestinal absorption, even for neutral amino acids, can be seen as partially a cause and partially a result of degrees of reluctance in making formal analog inhibition analyses for transport by this tissue. Hence unusual uncertainties tend to surround the identification of some of the intestinal brush-border transport systems as homogeneous entities. The problems surrounding the discrimination of intestinal transport systems for di- and tri-peptides $[15 a]$ are parallel but even more severe because of difficulties in access to the myriad of analogs likely to serve.

The strategy then is to proceed toward the ABC test by simplifying whatever kinetic complexities are encountered in the transport interaction between analogs. The devices available for simplification involve not only selection of test substrates and change of $\left[\mathrm{Na}^{+}\right]$, but also changes of $\left[\mathrm{H}^{+}\right]$. One may thus titrate the mediator to change its substrate selectivity, or one may titrate the amino acid to a different state of charge [7]. The test can be made instead under conditions that differentially regulate a transport system. The test can be made in the presence of respiratory inhibitors, sulfhydryl reagents, or other reagents that prove more or less system-specific. Antibodies specific to a critical component of a given transport system should become available. Unfortunately for amino acid transport we have not yet had access to an inhibitor as

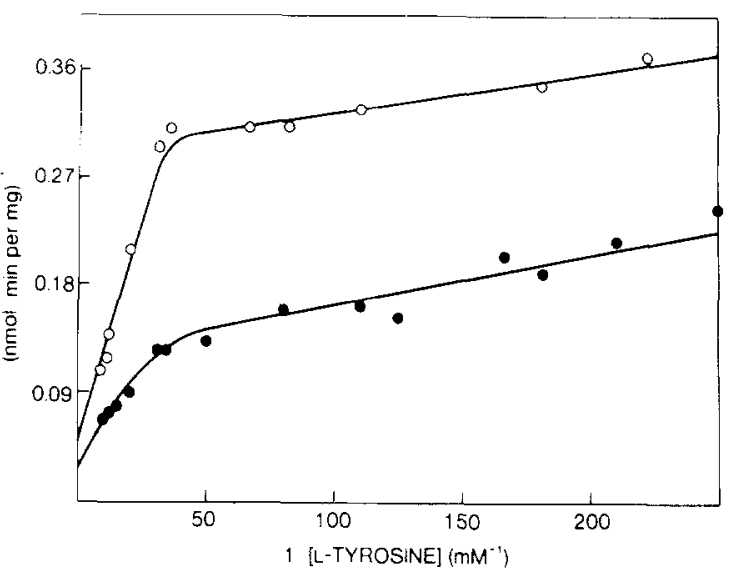

Fig. 5. A mutant of Bacillus subtilis with increased tyrosine uptake $(\Theta)$ retains the nonlinear kinetics shown by the parental wild type (O) [13]. Similar retention of the shape of the kinetic curve was obtained for a mutant showing decreased tyrosine transport. The Hill coefficients corresponding to the plots were not greatly changed on mutation

potent and selective so that it can serve in the way phloridzin serves for sugar transport. As an alternative approach, the transport measurements can be made over time intervals differentially favoring a slower or a faster system (e.g. as used in a step to discriminate System asc and L [ref. 22, Fig. 3a]), or at a substrate concentration favoring a system of higher or lower affinity (Fig. 6). The latter approach of course emphasizes that a study of the time course of solute uptake should be preliminary to any program of systems discrimination. Although the initial rate of entry is valuable as a measure of influx minimally contaminated by efflux, useful differentiations have nevertheless been obtained over longer time intervals.

\section{Concluding Comment}

The legend to Fig. 6 discusses as a summary illustration the delineation of the routes for glycine uptake into the hepatoma line HTC. This analysis began with the advantage that the $\mathrm{Na}^{+}$-dependent systems that were found to participate were already well known.

Although I should like to have succeeded in this brief presentation in describing here a sequential kinetic strategy that would serve to discriminate a suspected new transport system in any membrane context, it will be apparent that important gains in economy can be achieved by turning on occasion for a difficult discrimination to a more favorable tissue, cell or organelle in which the discrimination chances to be easier.

Until we know the gene that determines each membrane transport system, our list of distinct systems will be somewhat provisional. We hope the 


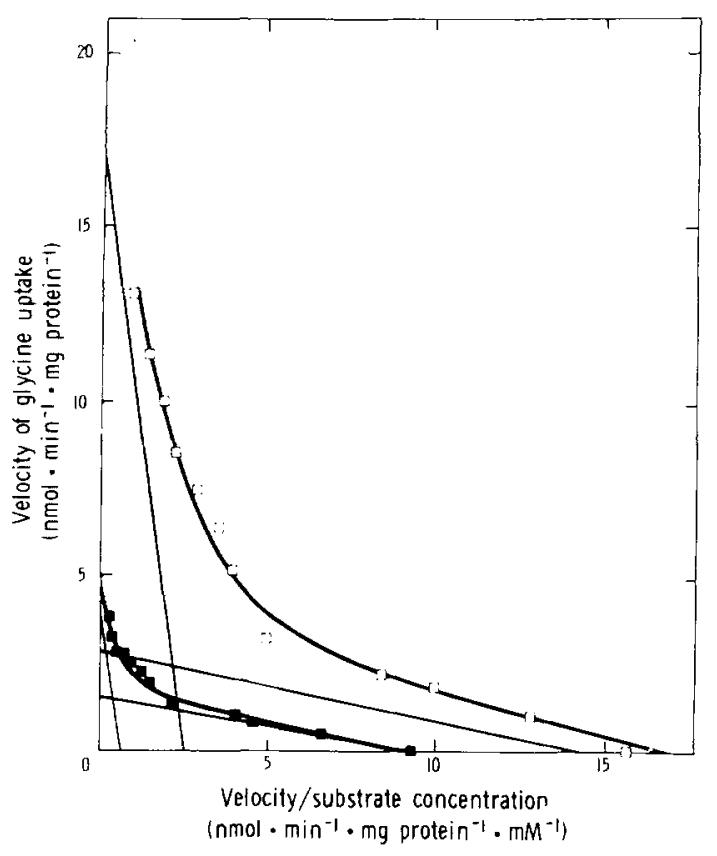

Fig. 6. Summary illustration of the discrimination of two transport systems for glycine uptake by the hepatoma cell line, HTC. The solid squares represent rates seen for cells first incubated 9 $\mathrm{hr}$ with dexamethasone, whereas the open squares are for cells held instead in the absence of this agent. The straight lines serve as in other studies to estimate the kinetic parameters applying for the two components of uptake, $K_{m}$ values $\cong 0.2$ and $5 \mathrm{~mm}$, respectively. The dexamethasone treatment reduced the $V_{\max }$ for the high-affinity component by $46 \%$ (see extrapolation to the ordinate), that for the low-affinity component by $72 \%$. These components of glycine uptake were also discriminated by their different sensitivities to lowering of the $\mathrm{pH}$, and to inhibition by various amino acid analogs. In most circumstances a deduction from each plotted observation of the uptake rate observed in the absence of $\mathrm{Na}^{+}$would have been wise. In the present instance, however, uptake in the absence of $\mathrm{Na}^{+}$proved negligible, both at $4 \mu \mathrm{M}$ and $6.3 \mathrm{mM}$ glycine. Reproduced with permission from S.B. Reichberg and T.D. Gelehrter (1980). J. Biol. Chem. 255:5710 (ref. [18] of this paper). A next stage of the discrimination showed by inhibition analysis that sarcosine serves as a model analog for the high-affinity component, and 2-(methylamino)isobutyric acid for the low-affinity component [9]. Furthermore the rate of glycine or sarcosine uptake by the high-affinity system of Fig. 6 was shown to bear a nearly linear relation to $\left[\mathrm{Na}^{+}\right]^{2}$ rather than to $\left[\mathrm{Na}^{+}\right][9]$, corresponding to a property seen for System Gly in pigeon erythrocytes and rabbit reticulocytes [23, 25]. These results indicated that System Gly produced the high-affinity glycine uptake also in HTC. (In our presentation [9] we unfortunately misstated that Reichberg and Gelehrter [18] had attributed this uptake to System ASC, whereas instead they attributed it to some previously uncharacterized transport system. We regret this mistake.) Both studies identified the low-affinity, highcapacity system of this figure (left) as System A

methods available will prove useful in the meantime for guiding that more ultimate identification of these systems.

Of the work reviewed here that derives from this laboratory, support is acknowledged from Grants HD01233 and AM 32281.
National Institutes of Health, United States Public Health Service.

\section{Glossary of Some Well-Characterized Transport Systems for Amino Acids}

For Zwitterionic Amino ACIDS (Note transport by these systems of basic and acidic amino acids when they are in zwitterionic form)

\section{Sodium-Dependent}

System A: $\quad$ Notably "uphill." Wide scope, but weaker for amino acids with branched or cyclic side chains. Various regulations converge on this system. Model substrate 2-(methylamino) isobutyric acid. (Note evidence for an Imino system more restricted to the prolines.)

System Gly: Glycine and sarcosine. Wide distribution. (Note iminoglycine system.)

System N: Glutamine, histidine and asparagine. Resemblance to System $A$ in sensitivity to adaptive regulation and $\mathrm{pH}$ sensitivity. Characterized for hepatocytes; distribution may be limited.

System ASC: Prefers 3 to 5 carbon atoms in a chain; does not tolerate an $\mathrm{N}$-methyl group. Ubiquitous and conspicuous; uphill action; responds to trans stimulation. On protonation, functions for $\mathrm{Na}^{+}$-dependent transport of anionic amino acids of similar chain length. (Note question as to identity of a possibly related brush-border system.)

Sodium-Independent (Designations for the first two systems listed below violate a recent convention that uses lowercase designations for $\mathrm{Na}^{+}$-independent transport systems [2].)

System L: Wide-scope, but prefers bulkier amino acids; hence bicyclic analogs have served as model substrates. Often only weakly concentrative and notably responsive to trans stimulation. Sometimes descriptions suffer from including contributions of other $\mathrm{Na}^{+}$independent systems; note designations $\mathrm{L} 1$, L2 in pointing out such a case [24].

System T: $\quad$ Selective for natural amino acids containing benzene rings [19], but also transports bicyclic amino acids. Distribution beyond human erythrocyte not well established. Weak response to trans stimulation; slow approach to steady state of uptake [22].

System asc: Selective for somewhat the same group of amino acids as $\mathrm{Na}^{+}$dependent System ASC, despite noninvolvement of $\mathrm{Na}^{+}$in selectivity for asc. Present in some mature mammalian red blood cells, although lacking in some phenotypes of the sheep [27] and the horse [12]. Presence in nucleated pigeon red cells and probable presence in rabbit reticulocytes points to a broad biological importance $[22]$. 


\section{For ANionic Amino Acids}

$\mathrm{Na}^{+}$-dependent systems are conventionally designated $\mathrm{X}$. $\mathrm{Na}^{+}$-independent systems $x$. Systems selective for aspartate and its anionic analogs of similar length are designated $\mathrm{x}_{\mathrm{A}}$ or $\mathrm{X}_{\mathrm{A}} ;$ systems selective for glutamate and its analogs are designated $x_{G}$ or $X_{i}$, unless cystine also serves as a substrate; in the latter case the designation $x_{C}$ is used. By this convention, the designation $\mathrm{X}_{A f i}$ is reserved for a $\mathrm{Na}^{+}$-dependent system not strongly selective for either aspartate or glutamate.

\section{For Cationic Amino Acids}

\section{Sodium-Independent}

System $y^{+}$: (Plus sign to indicate need for a charge on the side chain.) Diamino acids, arginine, cationic form of histidine. Ubiquitous, although more than one system may be involved. Directionality may be determined by the transmembrane potential. Certain neutral amino acids show $\mathrm{Na}^{+}$-dependent reactivity with $\mathrm{y}^{+}$. Shows strong trans stimulation. Ornithine system of hepatic mitochondria: unusual stereospecificity.

[Note the approach to a sequence $x, y, z$, in which $z$ is reserved for general use for systems for zwitterionic amino acids.]

\section{References}

1. Ahmed, K., Scholefield, P.G. 1962. Biochemical studies on l-aminocyclopentane carboxylic acid. Can. J. Biochem. Physiol. 40:1101-1110

2. Bannai, S., Christensen, H.N., Vadgama, J.V., Ellory, J.C., Englesberg, E., Guidotti, G.G., Gazzola, G.C., Kilberg, M.S., Lajtha, A., Sacktor, B., Sepúlveda, F.V., Young, J.D., Yudilevich, D., Mann, G. 1984. Amino acid transport systems. Nature (London) 311:308

3. Barry, P.H., Diamond, J.M. 1984. Effects of unstirred layers on membrane phenomena. Physiol. Rev. 64:763-872

4. Beyer, K.H., Wright, L.D., Skeggs, H.R., Russo, H.F., Shaner, G.A. 1947. Renal clearance of essential amino acids: Their competition for reabsorption by the renal tubules. Am. J. Physiol. 151:202-210

5. Christensen, H.N. 1964. Relations in the transport of $\beta$ alanine and the $\alpha$-amino acids in the Ehrlich cell. J. Biol. Chem. 239:3584-3589

6. Christensen, H.N. 1966. Methods for distinguishing amino acid transport systems of a given cell or tissue. Fed. Proc. 25:850-853

7. Christensen, H.N. 1984. Organic ion transport during seven decades: The amino acids. Biochim. Biophys. Acta 779:255269

8. Christensen, H.N., Handlogten, M.E. 1979. Interaction between parallel transport systems examined with tryptophan and related amino acids. J. Neural. Transm., Suppl. 15:1-13

9. Christensen, H.N., Handlogten, M.E. 1981. Role of System Gly in monolayer cultures of liver cells. Biochem. Biophys. Res. Commun. 98:102-107

10. Christensen, H.N., Liang, M., Archer, E.G. 1967. A distinct
$\mathrm{Na}^{+}$-requiring transport system for alanine, serine, cysteine, and similar amino acids. J. Biol. Chem. 242:5237-5246

11. Eavenson, E., Christensen, H,N. 1967. Transport systems for neutral amino acids in the pigeon erythrocyte. J. Biol. Chem. 242:5386-5396

12. Fincham. D.A., Masn, D.K., Young, J.D. 1985. Characterization of a novel $\mathrm{Na}^{+}$-independent ASC-like amino acid transponter in horse erythrocytes. Biochem. J. (in press)

13. Glover, G.E., D'Ambrosio, S.M., Jensen, R.A. 1975. Versatile properties of a nonsaturable, homogeneous transport system in Bacillus subtilis: Genetic, kinetic, and affinity labeling studies. Proc. Natl. Acad. Sci. USA 72:814-818

14. Kilberg, M.S., Handlogten, M.E., Christensen, H.N. 1980. Characteristics of an amino acid transport system in rat liver for glutamine, asparagine, histidine, and closely related analogs. J. Biol. Chem. 255:4011-4019

15. Kilberg, M.S., Handlogten, M.E., Christensen, H.N. 1981. Characteristics of System ASC for transport of neutral amino acids in the isolated rat hepatocyte. J. Biol. Chem. 256:3304-3312

15a. Matthews. D.M. 1984. Absorption of peptides, amino acids and their methylated derivatives. In: Aspartame: Physiology and Biochemistry. L.D. Stegink and L.J. Filer. Jr.. editors. pp. 29-46. Marcel Dekker, New York

16. Oxender, D.L., Christensen, H.N. 1963. Evidence for two types of mediation of neutral amino acid transport in Ehrlich cells. Nature (London) 197:765-767

17. Oxender, D.L., Christensen, H.N. 1963. Distinct mediating systems for the transport of neutral amino acids by the Ehrlich cell. J. Biol. Chem. 238:3686-3699

18. Reichberg, S.B., Gelehrter, T.D. 1980. Glucocorticoid inhibition of two discrete glycine transport systems in rat hepatoma cells. J. Biol. Chem. 255:5708-5714

19. Rosenberg, R., Young, J.D., Ellory, J.C. 1980. k-Tryptophan 1ransport in human red blood cells. Biochim. Biophys. Acta 598:374-384

20. Scriver, C.R., Wilson, O.H. 1964. Possible locations for a common gene product in membrane transport of imino-acids and glycine. Nature 202:92-93

21. Stevens, B.R., Ross, H.J., Wright, E.M. 1982. Multiple transport pathways for neutral amino acids in rabbit jejunal brush border vesicles. J. Membrane Biol. 66:213-225

22. Vadgama, J.V., Christensen, H.N. 1985. Discrimination of $\mathrm{Na}^{+}$-independent transport systems $\mathrm{L}, \mathrm{T}$, and asc in erythrocytes: $\mathrm{Na}^{+}$-independence of the latter a consequence of cell maturation? J. Biol. Chem. (in press)

23. Vidaver, G.A., Romain, L.F., Haurowitz, F. 1964. Some studies on the specificity of amino acid entry routes in pigeon erythrocytes. Arch. Biochem. Biophys. 107:82-87

24. Weissbach, L., Handlogten, M.E., Christensen, H.N., Kilberg, M.S. 1982. Evidence for two $\mathrm{Na}^{-}$-independent amino acid transport systems in primary cultures of rat hepatocytes. J. Biol. Chem. 257:12006-12011

25. Wheeler, K.P., Inui, Y., Hollenberg, P.F., Eavenson, E., Christensen, H.N. 1965. Relation of amino acid transport to sodium-ion concentration. Biochim. Biophys. Acta 109:620622

26. Wilbrandt, W., Rosenberg, T. 1961. The concept of carrier transport and its corollaries in pharmacology. Pharmacol. Rev. 13:109-183 (see pp. 117, 118, 125)

27. Young, J.D., Ellory, J.C., Tucker, E.M. 1975. Amino acid transport defect in glutathione-deficient sheep. Nature (London) 254:156-157

Received 6 September 1984 